\title{
Pacientes con infección por VIH/SIDA en una Unidad de Pacientes Críticos. La experiencia de un hospital general en un país en desarrollo
}

\author{
Fernando Verdugo, Francisco Pinto, Paulo Charpentier, Christian Von Mühlenbrock, \\ Andrés Soto, Jeannette Dabanch y Alberto Fica
}

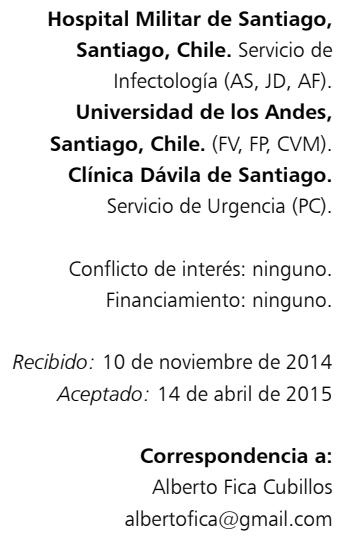

Hospital Militar de Santiago Santiago, Chile. Servicio de Infectología (AS, JD, AF). Universidad de los Andes, Santiago, Chile. (FV, FP, CVM) Clínica Dávila de Santiago. Servicio de Urgencia (PC)

Conflicto de interés: ninguno. Financiamiento: ninguno.

Recibido: 10 de noviembre de 2014 Aceptado: 14 de abril de 2015

Correspondencia a: Alberto Fica Cubillos albertofica@gmail.com

\section{HIV/AIDS patients in a Critical Care Unit. The experience of a general hospital in a developing country}

Introduction and aims: Despite general availability of HAART in Chile, admissions of HIV/AIDS patients to Intensive-Intermediate Care Units (IICU) are still happening, and a characterization of patient's profile, mortality and potentially avoidable admissions is necessary. Methods: Observational retrospective study in one general hospital in Chile of HIV/AIDS patients admitted to IICU during 9 years. Results: During 2005-2013, 32 patients were admitted to IICU, with $87.5 \%$ in AIDS stage, only $53.1 \%$ knew his/her condition, $43,8 \%$ were receiving HAART and $16.6 \%$ chemoprophylaxis for opportunistic infections. A CD4 count $<200 / \mu \mathrm{L}$ was registered in $75.9 \%$ of patients. Most admissions were driven by infectious conditions (84.4\%) and 48.1\% developed septic shock. IICU hospitalizations were motivated by respiratory failure, neurologic compromise, sepsis or a mixture of them $(87.5 \%)$. By univariate analysis, admissions by respiratory failure were associated to no HAART, oral candidiasis or CD4 $<250 / \mu \mathrm{L}(\mathrm{p}<0.01)$. Eight patients died during their first hospitalization $(25 \%)$ and other 5 in the following month after discharge. Death during hospitalization was significantly associated to vasoactive drug use $\geq 7$ days (OR 16.5; IC95 2.1-128 p < 0.01). In multivariate analysis, APACHE score $\geq 18$ was associated with death during hospitalization of after discharge (OR 3.3 IC95 1.1-10; p < 0.05). Four patients $(12.5 \%)$ had potentially avoidable admissions. Conclusions: Despite HAART availability in Chile, hospitalizations of patients with HIV/AIDS are still happening, affecting those that either are unaware of his/her condition, are not receiving HAART and/or chemoprophylaxis. These admissions generate premature deaths and happen even after discharge in severely ill patients.

Key words: HIV, acquired immunodeficiency syndrome, intensive care, in-hospital mortality, risk factors, respiratory failure, severe sepsis, septic shock, highly active antiretroviral therapy, long term survivors, adult.

Palabras clave: VIH, Síndrome de inmunodeficiencia adquirida, cuidados intensivos, mortalidad hospitalaria, factores de riesgo, falla respiratoria, sepsis grave, shock séptico, terapia antirretroviral altamente activa, sobreviviente a largo plazo, adulto.

\section{Introducción}

A pesar del advenimiento del tratamiento antiretroviral de gran actividad (TARGA), los ingresos de pacientes con infección por VIH a la UCI siguen ocurriendo e incluso aumentando y la mortalidad se mantiene ${ }^{1,2}$. Ello ocurre al parecer por problemas de acceso o por una infección por VIH avanzada no diagnosticada. La mortalidad aproximada de estos pacientes en UCI es de $25 \%$ y está asociada a aquellos pacientes que presentan antecedentes de sarcoma de Kaposi, recuento $\mathrm{CD} 4 \leq 50 / \mathrm{mm}^{3}$, mayor gravedad al ingreso, pobre estado funcional, necesidad de ventilación mecánica y falla cardíaca o uso de fármacos vasoactivos ${ }^{1,3}$. La mayor parte de los ingresos a UCI está motivada por enfermedades infecciosas (70-80\%), incluyendo neumonía bacteriana o por Pneumocystis jiroveci, neumotórax asociado a $P$. jiroveci, tuberculosis y toxoplasmosis cerebral ${ }^{1}$. A pesar del mal pronóstico inicial, la mortalidad a tres meses en los sobrevivientes ha mejorado con TARGA respecto a series históricas que no contaban con esta alternativa terapéutica (6 versus $15 \%$ ) lo que subraya la importancia de combinar las terapias en UCI con el tratamiento de la infección por VIH/SIDA. Además, de los ingresos a UCI, los pacientes con infección por VIH o SIDA experimentan complicaciones propias de su edad o de aquellas facilitadas por las alteraciones metabólicas asociadas, determinando también ingresos o Unidades Coronarias Intensivas ${ }^{4}$.

A pesar de la importancia de la infección por VIH en Chile, existe escasa información sobre el perfil de pacien- 
tes que ingresan a Unidades de Pacientes Críticos (UPC: Intensivo o Sala Intermedia), las causas que explican estos ingresos y su evolución. Los pocos datos que existen se limitan a reporte de casos, patologías específicas y una pequeña serie de 11 pacientes en ventilación mecánica ${ }^{5-8}$. El poder contar con esta información es relevante para demostrar el pronóstico adecuado que muchos de ellos tienen y justificar así el acceso a una medicina de alta complejidad. Es importante también evaluar el volumen de pacientes que ingresa sin diagnóstico previo de infección por VIH para estimular las acciones de pesquisa precoz antes de una complicación avanzada y conocer el porcentaje de ingresos evitables. Para contestar estas interrogantes, se efectuó un estudio observacional retrospectivo en pacientes que ingresaron a la UPC en el Hospital Militar de Santiago durante nueve años.

\section{Pacientes y Métodos}

Los pacientes fueron identificados por el registro de infectados por VIH que mantiene el Servicio de Infectología desde el año 2005 y que han sido hospitalizados en la UPC (Unidad de Cuidados Intensivos [UCI], Unidad Coronaria [UCO] o Sala de Cuidados Intermedios [UTI]) del Hospital Militar de Santiago, una institución que atiende a funcionarios activos o en retiro, sus cargas familiares y que también presta atención a pacientes externos. Sólo se incluyeron pacientes adultos durante su primera hospitalización en este tipo de unidades para evitar la distorsión que generan las re-hospitalizaciones por la misma causa o por una condición avanzada de la enfermedad. Salvo las re-hospitalizaciones y la edad, no hubo criterios de exclusión.

Los objetivos específicos del trabajo fueron establecer las características demográficas de estos pacientes, medidas preventivas en uso, sus manifestaciones clínicas, causas de ingreso a UPC, etapa clínica de la infección por $\mathrm{VIH}$, agentes infecciosos involucrados, complicaciones, duración de la estadía hospitalaria, condición al alta y al seguimiento post egreso.

En forma retrospectiva, se analizaron variables demográficas, co-morbilidades, si la condición de infección por VIH o SIDA era conocida o no antes del ingreso, uso de quimioprofilaxis para infecciones oportunistas o tuberculosis, antecedentes de vacunas para Streptococcus pneumoniae e influenza, manifestaciones clínicas, carga viral y recuento de linfocitos T CD4 si estuvieran disponibles, diagnósticos asociados al ingreso, agentes infecciosos involucrados, tratamientos recibidos, complicaciones durante la evolución, incluyendo efectos adversos a medicamentos y condición al egreso.

Definiciones. Para las categorías de sepsis, sepsis grave y shock séptico se aplicaron definiciones internacionales ${ }^{9}$.
La presencia de falla respiratoria se definió por la presencia de una relación $\mathrm{PaFiO}_{2}<300, \mathrm{PaO}_{2}<60 \mathrm{mmHg}$, saturación de $\mathrm{O}_{2}<90 \%$ con aire ambiental o conexión a ventilación mecánica. Se consideró un caso con toxoplasmosis si presentó lesiones cerebrales compatibles asociadas a una respuesta positiva al tratamiento con cotrimoxazol y/o serología IgG positiva para Toxoplasma gondii. Los casos de neumonía por $P$. jiroveci fueron asignados como tal si fueron confirmados por algún método microbiológico. De la misma manera, los casos asignados como enfermedad por CMV fueron definidos por un cuadro clínico compatible asociado a una prueba histológica compatible, test positivo en sangre (RCP o antigenemia) o por exudados característicos al examen del fondo de ojo.

Para la extracción de los datos se confeccionó una plantilla especial y análisis con el programa SPSS v15.

El trabajo fue autorizado por el Comité de Ética del Hospital Militar de Santiago.

Análisis estadístico. Los datos se presentan de una manera descriptiva con algunas variables categorizadas. Como medida de tendencia central se usó la mediana y de dispersión el rango interquartil 25-75\% (RIQ 25-75). Se analizaron factores asociados a ingreso a UCI respecto a los que ingresaron a intermedio o un desenlace fatal mediante un análisis bivariado con la prueba de $\chi^{2}$ o de Fisher con el respectivo cálculo del odds ratio (OR). La condición (vivo o fallecido) a largo plazo se determinó mediante la búsqueda de certificados de defunción en mayo de 2014 en la página del Servicio de Registro Civil e Identificación de Chile (http://www.registrocivil. cl). Para el seguimiento a largo plazo se aplicaron curva de sobrevida de Kaplan Meier y regresión de Cox con Log Rank test para contraste de hipótesis con diferentes variables. Se aplicaron pruebas no paramétricas en casos con bajo número de datos (prueba bilateral de Fisher para tablas de contingencias de variables categóricas o prueba de Mann-Whitney para comparar variables continuas entre diferentes grupos). Se consideró un valor $\mathrm{p}<0,05$ como estadísticamente significativo.

\section{Resulltados}

Un total de 32 pacientes que ingresaron a UPC por primera vez fue identificado entre los años 2005 y 2013 con un rango de 1 a 11 hospitalizaciones por año sin observar un incremento o disminución de admisiones en el tiempo. Once casos ingresaron a Intermedio $(34,4 \%)$ y el resto a UCI-Coronaria (n: $21 ; 65,6 \%$ ). El 75\% de los pacientes era de sexo masculino (n: 24). La mediana de edad fue de 40 años (rango 20-80) con $81,3 \%<60$ años. La prevalencia declarada de bebedores excesivos y usuarios de drogas ilícitas fue baja $(<10 \%)$, no así la de 
Tabla 1. Co-morbilidades, hábitos y co-infecciones virales en 32 pacientes en hospitalización inicial en unidades críticas o semicríticas

\begin{tabular}{lcc|} 
Antecedente & $\mathbf{n}$ & $\%$ \\
Tabaquismo (cualquier consumo) & 10 & 32,3 \\
Bebedor excesivo & 3 & 9,4 \\
Usuario de drogas ilícitas & 2 & 6,5 \\
Diabetes mellitus & 3 & 9,4 \\
Hepatopatía & 3 & 9,4 \\
Cardiopatía & 1 & 3,1 \\
Co-infección por VHB & 2 & 6,3 \\
Co-infección por VHC & 1 & 3,1 \\
Sífilis (latente) & 1 & 3,1 \\
\hline
\end{tabular}

Tabla 2. Recuento de linfocitos, CD4 y carga viral en pacientes con y sin TARGA al ingreso

\begin{tabular}{lcccccc} 
& \multicolumn{4}{c}{ Sin TARGA } & \multicolumn{3}{c}{ Con TARGA } \\
Parámetro & $\mathbf{n}$ & Mediana & RIQ 25-75 & $\mathbf{n}$ & Mediana & RIQ 25-75 \\
Linfocitos/mm & 25 & 530 & $190-4.658$ & 7 & 1.036 & $646-1.480$ \\
CD4/mm & 23 & $21,2^{*}$ & $2-799$ & 6 & $247,5^{*}$ & $28-795$ \\
Carga viral/mL Log 10 & 16 & 5,2 & $4,9-6,0$ & 4 & 5,3 & $0,0-5,6$
\end{tabular}

${ }^{*} p<0,05$ por Prueba no paramétrica de Mann-Whitney. TARGA: tratamiento anti-retroviral de gran actividad.

\section{Tabla 3. Causa primaria de hospitalización}

\section{Causa primaria}

Infecciosas

Pulmonar

Neumonía por Pneumocystis jiroveci

Tuberculosis pulmonar

Neumonía por CMV

Neumonía neumocóccica

Neumonía sin agente identificado

\section{SNC}

Toxoplasmosis cerebral

Meningitis aguda a líquido claro

\section{Bacteriemias}

S. aureus

E. coli

\section{Mixtas}

Toxoplasmosis cerebral y neumonía

Abscesos cerebrales y neumonía

Neumonía y colangitis aguda bacteriana

Neumonía y abscesos hepáticos

Otras

Peritonitis bacteriana espontánea

Malaria grave por Plasmodium falciparum

No infecciosas

Hemorragia digestiva alta por várices esofágicas Hematoma retroperitoneal espontáneo

Síndrome Guillain-Barré

Síncope por enfermedad del nodo sinusal

Trombo-embolismo pulmonar

\section{n (\%) Comentarios}

$27(84,4 \%)$

$13(40,6 \%)$

$7(21,9 \%) \quad$ Un caso asociado a neutropenia febril

$1(3,1 \%)$

$1(3,1 \%)$

$1(3,1 \%) \quad$ Con bacteriemia asociada

$3(9,4 \%)$

$5(15,6 \%)$

$2(6,3 \%)$

$3(9,4 \%)$ Primoinfección (2) y primoinfección asociado a meningitis por enterovirus (1)

$2(6,3 \%)$

$1(3,1 \%) \quad$ Con focos embólicos pulmonares

$1(3,1 \%)$ Secundaria a foco renal con litiasis

$5(15,6 \%)$

$2(6,3 \%)$

$1(3,1 \%)$

$1(3,1 \%) \quad$ Colangitis esclerosante previa con stent

$1(3,1 \%)$

$2(6,3 \%)$

$1(3,1 \%) \quad$ Cirrosis por virus hepatitis $C$

$1(3,1 \%) \quad$ Adquirida en Guinea Ecuatorial

$5(15,6 \%)$

$1(3,1 \%) \quad$ Paciente con cirrosis criptogénica

$1(3,1 \%)$ Linfoma no Hodgkin (debut)

$1(3,1 \%)$

$1(3,1 \%)$

$1(3,1 \%)$ Paciente postrada en su casa tabaquismo que llegó a un tercio (Tabla 1). De la misma manera, la prevalencia de co-infecciones (hepatitis B y C) fue infrecuente al igual que de co-morbilidades como diabetes mellitus y daño hepático crónico.

La mayor parte de los casos estaba en etapa C3 $(62,5 \%)$ y 28 pacientes $(87,5 \%)$ estaban en etapa SIDA según definición del CDC (A3, B3 o C1-3). Los valores para otras etapas fueron A1 (1 caso; 3,1\%), A2 (2 casos; 6,3\%) y B1 (1 caso; $3,1 \%$ ).

Sólo 53,1\% (n: 17) de los pacientes conocían el diagnóstico de infección por VIH al momento de la hospitalización. De los 17 pacientes con diagnóstico conocido, 16 tenían indicación de recibir terapia anti-retroviral (TARGA) pero sólo 7 de ellos (43,8\% del subgrupo con diagnóstico conocido y $21,9 \%$ del total) la estaba recibiendo. La falta de tratamiento se distribuyó homogéneamente entre los pacientes entre el año 2005 y 2013, no apreciándose que esta falencia se concentrara sólo en los primeros años de este registro cuando el acceso universal recién comenzaba. Un paciente había suspendido la TARGA $(5,9 \%)$.

Sólo un paciente de los 17 con infección por VIH conocida se encontraba vacunado contra la hepatitis B (5,9\%), dos contra la influenza correspondiente al año $(11,8 \%)$ y ninguno contra $S$. pneumoniae. De los 17 pacientes con diagnóstico de infección por VIH conocido, 12 pacientes requerían profilaxis con cotrimoxazol pero sólo dos la estaban recibiendo (16,6\% de este subgrupo).

La mediana de linfocitos totales al ingreso en la primera hospitalización fue de 673/ $/ \mathrm{mm}^{3}$ (RIQ 25-75: 425 1034) con $65,6 \%<1.000 / \mathrm{mm}^{3}$. Se contó con recuento de linfocitos CD4 en 29 pacientes que demostró una mediana de $28 / \mathrm{mm}^{3}$ (rango 2-799) con $75,9 \%<200 / \mathrm{mm}^{3}$. El análisis por subgrupos reveló un recuento significativamente inferior de linfocitos CD4 en el subgrupo sin TARGA $(p<0,05)$ y una tendencia a una mayor carga viral en el grupo sin TARGA (p: 0,053) (Tabla 2). Las diferencias en el recuento de linfocitos totales no fueron significativas. La mediana de la carga viral en 23 pacientes sin TARGA con datos disponibles fue de $\log 105,3$ copias por $\mathrm{mL}$ (RIQ 25-75: $\log 10$ 4,9-6,0).

La mayoría de los ingresos fue motivado por una causa infecciosa (n: 27; 84,4\%) (Tabla 3). Dentro de las causas infecciosas, las infecciones pulmonares representaron $40 \%$ de los casos, secundadas por patología infecciosa del SNC e infecciones mixtas. Otras causas infecciosas primarias estuvieron representadas por eventos de bacteriemia, incluyendo casos de malaria importada y peritonitis bacteriana espontánea.

En conjunto, las infecciones pulmonares, fuese en procesos aislados o mixtas, estuvieron presentes en cerca de la mitad de los casos (n: 18; 56,3\%). De la misma manera, de los cinco casos con toxoplasmosis cerebral o abscesos cerebrales, tres de ellos se presentaron además 


\begin{tabular}{|c|c|c|c|c|c|c|}
\hline \multirow[t]{2}{*}{ Causa } & \multicolumn{2}{|c|}{ UTI* } & \multicolumn{2}{|c|}{ UCI/UCO** } & \multicolumn{2}{|c|}{ Global } \\
\hline & $\mathbf{n}$ & $\%$ & $\mathbf{n}$ & $\%$ & $\mathbf{n}$ & $\%$ \\
\hline Insuficiencia respiratoria & 4 & 36,4 & 4 & 19,0 & 8 & 25,0 \\
\hline Insuficiencia respiratoria y sepsis grave & 0 & 0,0 & 7 & 33,3 & 7 & 21,9 \\
\hline Compromiso neurológico & 4 & 36,4 & 4 & 19,0 & 8 & 25,0 \\
\hline Sepsis grave o shock séptico & 0 & 0,0 & 4 & 19,0 & 4 & 12,5 \\
\hline Insuficiencia respiratoria, sepsis y compromiso neurológico & 0 & 0,0 & 1 & 4,8 & 1 & 3,1 \\
\hline Evento cardiovascular & 0 & 0,0 & 1 & 4,8 & 1 & 3,1 \\
\hline Otros & $3^{* * *}$ & 27,3 & 0 & 0,0 & 3 & 9,4 \\
\hline Total & 11 & 100,0 & 21 & 100,0 & 32 & 100,0 \\
\hline
\end{tabular}

Tabla 5. Distribución por causa principal de ingreso en 32 pacientes con o sin TARGA en hospitalización en UPC

\begin{tabular}{|lccc|}
\hline Condición & Con TARGA & Sin TARGA & Total \\
Insuficiencia respiratoria aislada o asociada a otras condiciones & $0^{*}$ & $16^{*}$ & 16 \\
Sepsis grave sin otras condiciones & 1 & 3 & 4 \\
Neurológico & 2 & 6 & 8 \\
Cardiovascular & 1 & 0 & 1 \\
Otro & 3 & 0 & 3 \\
Total & 7 & 25 & 32 \\
\hline *Diferencia significativa, $p<0,01$. & & & \\
\hline
\end{tabular}

con neumonía. Por otra parte, al menos tres eventos de meningitis motivaron el ingreso a unidades críticas o semi-críticas y todas ellas fueron compatibles con infección retroviral primaria o co-infección en un caso con enterovirus. No se encontraron en esta serie casos de meningitis por Cryptococcus neoformans o infecciones por micobacterias atípicas.

Además de las infecciones descritas como causa primaria de hospitalización, se registraron cuatro casos con esofagitis por Candida (12,5\%) y dos infecciones por CMV (retinitis e infección ulcerativa gastrointestinal).

De los pacientes que ingresaron por infección, $92,6 \%$ (n: 25) manifestaron criterios de sepsis, 63\% (n: 17) de sepsis grave y 48,1\% (n: 13) de shock séptico.

En la Tabla 4 se presentan las principales causa de ingreso a UPC. Se puede apreciar que la insuficiencia respiratoria y el compromiso neurológico fueron causas importantes de ingreso y que la combinación de insuficiencia respiratoria y sepsis grave o shock séptico fue relevante en esta serie. Además, se observaron tres causas misceláneas correspondientes a un evento de hemorragia digestiva alta por varices esofágicas, un caso de hematoma retroperitoneal espontáneo y un tercer caso por peritonitis bacteriana espontánea en una paciente con cirrosis hepática por virus hepatitis $\mathrm{C}$, todos hospitalizados en Unidad Intermedia. El bajo tamaño de la muestra impidió detectar diferencias significativas para estas causas entre las unidades de hospitalización.

Impacto de TARGA, candidiasis oral y los recuentos de linfocitos en la causa de ingreso a UPC (Tabla 5). Los pacientes que presentaron falla respiratoria como causa principal de ingreso a UPC, fuese aislada o asociada a otras condiciones, se asociaron significativamente a la ausencia de TARGA ( $<<0,01$; OR 26 IC95 1,3-508). Por otra parte, 12 de los 15 pacientes con candidiasis oral presentaron insuficiencia respiratoria como causa principal o asociada de ingreso versus cuatro de los 17 sin candidiasis oral ( $p<0,01$, OR 13 IC $952,4-70)$. Además, un recuento de linfocitos $\mathrm{CD} 4<250 / \mathrm{mm}^{3}$ se asoció significativamente al ingreso a UPC por insuficiencia respiratoria aislada o asociada ( $p<0,01$ OR 25,6 IC 95 1,3-506). Finalmente, el diagnóstico confirmado de neumonía por $P$. jiroveci se asoció significativamente al ingreso a UPC por falla respiratoria aislada o combinada. 
Todos los pacientes con este agente (n: 7) presentaron falla respiratoria versus nueve de los 25 en los que no se estudió o no se documentó este agente $(\mathrm{p}<0,01$; OR 26 IC 95 1,3-508). Debido al bajo número de pacientes no se pudo hacer un análisis multivariado.

Manifestaciones clínicas al ingreso. En la Tabla 6 se presentan las manifestaciones clínicas detectadas al ingreso donde destacan por su frecuencia, la presencia de fiebre, hipotensión arterial, manifestaciones neurológicas, desnutrición, candidiasis oral y signos pulmonares. No se detectaron pacientes con lesiones genitales o sarcoma de Kaposi cutáneo.

Pacientes que ingresaron a UCI. De los 20 pacientes que ingresaron a UCI en la primera hospitalización, 18 $(85,7 \%)$ requirieron ventilación mecánica y 17 (81\%) fármacos vaso-activos; estas segundas fueron requeridas no sólo por los pacientes con shock séptico sino que también en aquellos con hipertensión endocraneal, shock cardiogénico y vasoplejia asociada a polineuropatía tipo síndrome de Guillain-Barré.

Score de gravedad al ingreso. Los pacientes que ingresaron a UCI/UCO tuvieron en promedio un valor APACHE II de 18,4 (rango 8-29) y los de UTI un promedio de 14,3 (rango 6-23). Las diferencias no fueron significativas. Tampoco se encontraron diferencias significativas entre el grupo con TARGA versus el grupo sin TARGA al ingreso (datos no mostrados).

Pacientes que ingresaron a Intermedio. Once pacientes ingresaron a Intermedio por causas respiratorias, neurológicas u otras condiciones (Tabla 4). Tres de los cuatro pacientes que ingresaron por causa respiratoria lo hicieron por neumonía por $P$. jiroveci confirmada o presunta y uno por trombo-embolismo pulmonar. Ninguno de ellos requirió ventilación no invasora. A su vez, los cuatro pacientes que ingresaron por condiciones neurológicas lo hicieron por meningoencefalitis (n: 2) o lesiones cerebrales (n: 2).

Evolución. La estadía hospitalaria promedio fue de 20,4 días (RIQ 25-75: 9 a 24,5, mediana 16,5). La estadía de los pacientes en UCI fue en promedio de 24 días (mediana 17 días) y de 13,6 días en aquellos que se hospitalizaron en intermedio (mediana 13 días) pero esta diferencia no fue significativa. En ningún caso se estableció una limitación del esfuerzo terapéutico. La suma de días camas ocupadas en intermedio alcanzó los 149 días, equivalente a $29,6 \%$ de la suma de días camas en UCI (504 días).

Cirugía o procedimientos quirúrgicos y otras intervenciones. Dos pacientes de la serie $(6,3 \%)$ fueron sometidos a cirugía en su primera hospitalización, correspondientes en un caso a la instalación de un marcapasos y en otro a la resección segmentaria pulmonar por fístula bronco-pleural en un paciente con neumonía viral y neumotórax al ingreso. Adicionalmente, un paciente $(4,9 \%)$ requirió diálisis.

\begin{tabular}{|lcc|}
\hline \multicolumn{3}{|c}{ Tabla 6. Manifestaciones clínicas al ingreso } \\
(no excluyentes)
\end{tabular}

Tratamiento anti-retroviral en UPC. En siete casos se inició TARGA $(21,9 \%)$ durante la estadía hospitalaria, decisión que quedó supeditada a criterio del equipo tratante. Cuatro de estos pacientes fallecieron en la misma hospitalización y uno de ellos tuvo una complicación letal, probablemente asociada al inicio de TARGA (ver más adelante). Estos cuatro pacientes fallecieron entre $0 \mathrm{y}$ 3 días desde el inicio del TARGA y otro paciente falleció pocos días después del alta a los 44 días de iniciar el tratamiento. En seis pacientes se inició abacavir-lamivudina con efavirenz. En cuatro de ellos se hizo evaluación farmacogenómica previa sobre la presencia del alelo HLA B*5701 que demostró la ausencia de este rasgo y para los otros dos casos con uso de abacavir, este examen no estuvo disponible. En un caso con síndrome de Guillain Barré se utilizó zidovudina-lamivudina con ritonavir/ lopinavir co-formulado sin estar recibiendo medicamentos contraindicados en ese momento.

Mortalidad. Ocho pacientes fallecieron en la primera hospitalización (25\%). Todos los decesos salvo uno, ocurrieron en UCI. La mortalidad para los pacientes que ingresaron a UCI fue de $33,3 \%$ ( 7 de 21 ) y de $9,1 \%$ para aquellos en UTI (1 de 11). El desenlace fatal sólo estuvo significativamente asociado al uso de fármacos vasoactivos por siete o más días (OR 16,5; IC 95 2,1-128 p <0,01). La mortalidad no estuvo asociada a otros factores como la presencia de SIDA, el recuento de linfocitos CD4, el uso previo de TARGA, la presencia o no de infección, shock séptico, score de gravedad, ventilación mecánica, año de ingreso o intervalo entre inicio de síntomas e ingreso. 
Mortalidad a largo plazo. Hasta el cierre de este reporte, cinco pacientes habían fallecido además de los ocho durante la hospitalización estudiada. En el análisis estratificado, una edad $>50$ años $(<0,05)$ y un puntaje APACHE II $\geq 18$ puntos ( $<0,05)$, se asociaron significativamente a una menor sobrevida hasta un y medio año post-alta. En el análisis multivariado con regresión de Cox, sólo un score APACHE $\geq 18$ se asoció en forma independiente a este desenlace (OR 3,3 IC 95 1,1-10; $\mathrm{p}<0,05)$ (Figura 1).

La causa de muerte estuvo relacionada al motivo de ingreso en tres de los ocho decesos pero tuvo una causa diferente en los cinco casos restantes e incluyó un evento de shock anafiláctico probablemente asociado a TARGA en un paciente con estudio genotípico negativo para HLA B*5701 (ver discusión). Todos salvo uno, fallecieron antes de cumplir su expectativa de vida indicando muertes prematuras.

Hospitalizaciones potencialmente evitables. Cuatro pacientes $(12,5 \%)$ con diagnóstico conocido de infección por VIH y sin tratamiento anti-retroviral y/o quimioprofilaxis, tuvieron hospitalizaciones potencialmente evitables (dos casos con toxoplasmosis cerebral y dos eventos demostrados de neumonía por $P$. jiroveci, respectivamente).

\section{Discusión}

La información publicada sobre la evolución de pacientes con infección por VIH hospitalizados en UCI en Chile es escasa. Hasta la fecha, sólo se cuenta con un reporte que describe el curso de 11 pacientes sin TARGA observados en el período 2000-2004 conectados a ventilación mecánica y con una tasa de mortalidad de $63 \%{ }^{8}$. El análisis de un mayor número de casos en años recientes es necesario para evaluar si las cifras de mortalidad han disminuido con la generalización en el acceso a la TARGA desde el $2004^{10}$, estimar la magnitud del uso de recursos críticos y semi-críticos, identificar factores de riesgo asociados a un desenlace fatal, estimar el porcentaje de hospitalizaciones potencialmente prevenibles y conocer la proporción de casos que ingresan sin diagnóstico previo. Además, es relevante conocer los agentes infecciosos y causas no infecciosas en la era TARGA para evaluar la posible emergencia de nuevos patógenos y el peso que pueden tener las intervenciones preventivas mediante quimioprofilaxis y vacunas.

Varios hallazgos de este trabajo merecen ser comentados. Por ejemplo, se pudo objetivar que los ingresos a UPC siguen ocurriendo a pesar de la facilidad del acceso a TARGA en Chile y que el perfil de pacientes corresponde a uno en etapas avanzadas de enfermedad que frecuentemente no conoce su condición o que a pesar de conocer su diagnóstico, no está en tratamiento.

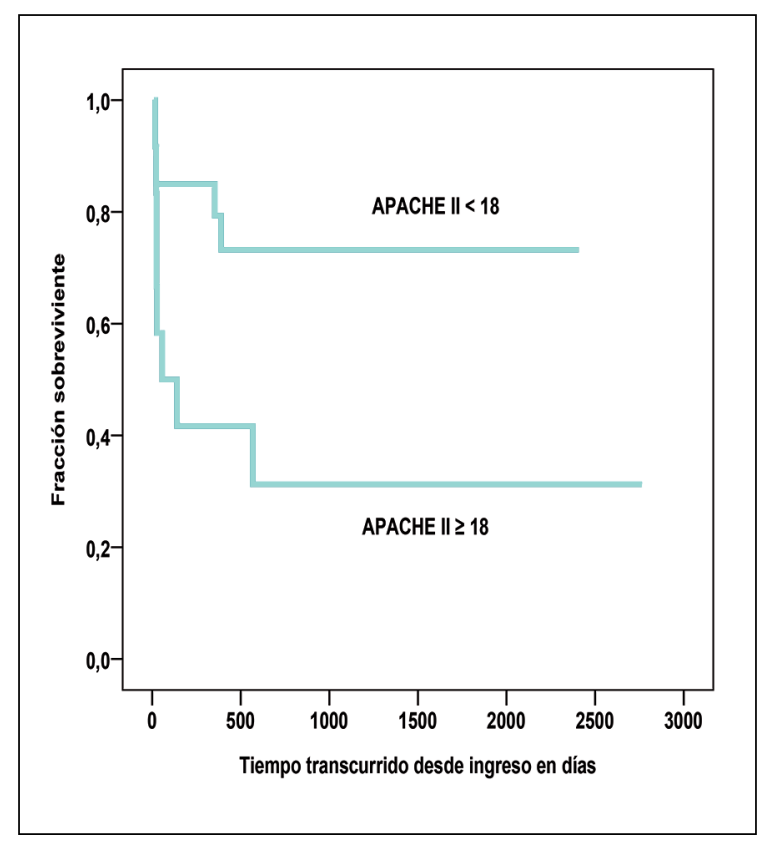

Figura 1. Sobrevida según puntaje APACHE II de gravedad al ingreso en un grupo de pacientes hospitalizados en unidades críticas o semicríticas con infección por VIH/SIDA. Hospital Militar de Santiago, 2005-2013.
En esta serie, el porcentaje de pacientes con infección en etapa SIDA supera el $85 \%$ de los casos, una cifra similar a otros reportes en Latinoamérica ${ }^{11}$, lo que ilustra las dificultades para efectuar un tamizaje diagnóstico inicial y de accesibilidad a la terapia antes de la aparición del SIDA. Debido a que en Chile el acceso a la terapia con cobertura financiera está asegurado, son necesariamente otros las determinantes que impiden a aquellos con el diagnóstico de infección por VIH previo, acercarse al sistema de salud para iniciar intervenciones de alto beneficio. Aunque no estudiados en detalle en nuestro medio, información proveniente de otros países indica que las barreras para el diagnóstico y atención de estos pacientes están asociadas a la pertenencia a ciertas etnias, promiscuidad sexual, alta frecuencia de rechazo al test en el medio, ciertos grupos de edad, no uso de preservativos o miedo al estigma ${ }^{12}$. Son necesarios mayores estudios y campañas destinadas al manejo de estas barreras para evitar estos ingresos en la era de un fácil acceso a TARGA. De acuerdo a nuestros resultados, al menos $12 \%$ de las hospitalizaciones observadas podrían tal vez haberse evitado con un acceso más oportuno a TARGA o profilaxis de infecciones oportunistas. Sin embargo, es probable que el impacto pueda ser mayor debido al beneficio transversal que tiene la corrección de la inmunodeficiencia a través del tratamiento. Los datos comentados indican un gran desafío a la salud pública y la necesidad de crear nuevas campañas, esta vez para fomentar el control de aquellos con diagnóstico conocido.

Otros hechos identificados en este trabajo incluyen la transversalidad de género aunque con una mayor carga 
para el sexo masculino y el gran impacto en mortalidad sobre personas en etapa laboralmente productiva. La descripción de mujeres hospitalizadas en UCI con infección por VIH ya ha sido reportada en nuestro país ${ }^{8}$. Por su parte, la mortalidad hospitalaria global fue elevada (25\%), especialmente en el grupo en UCI $(33,3 \%)$, cifra algo diferente y aparentemente inferior a lo reportado previamente en Chile $(63 \%)$ y Brasil $(46 \%)^{8,11}$. La elevada letalidad se combina con una muerte prematura contribuyendo a la carga de enfermedad que tiene esta patología. Hacia el año 2004, la carga de enfermedad por infección VIH y SIDA representaba $\sim 9.000$ años de vida perdidos ajustados por discapacidad (AVISA) y ocupaba el lugar número 15 por este concepto en hombres entre 20 y 44 años y en forma más específica, esta patología ocupaba el primer lugar por enfermedades trasmisibles en hombres en esa época ${ }^{13}$. En nuestro trabajo no se evaluó una discapacidad permanente que también contribuye a la carga de enfermedad medida por AVISA y que es un hecho conocido entre los sobrevivientes de $\mathrm{UCI}^{14}$.

La ocupación de camas intermedias no es despreciable y representa cerca un tercio de la ocupación en días-camas respecto a los requerimientos de UCI, enfatizando la demanda de recursos hospitalarios que representa la infección VIH-SIDA. No tenemos conocimiento de otras evaluaciones sobre este aspecto.

A pesar del promedio etario joven de estos pacientes, se observa una fracción de la población con co-morbilidades crónicas o hábito tabáquico, lo que es relevante como factor de riesgo de enfermedades cardiovasculares en pacientes que sobreviven por largos años debido a la terapia, la que se suma a los cambios metabólicos y trastornos vasculares. El tabaquismo impacta también en el riesgo de infecciones pulmonares complicadas o de cáncer y el alcoholismo en el riesgo de infecciones neumocóccicas, hepatoxicidad por fármacos o progresión de co-infección por virus hepatotropos. En algunos casos, estas co-morbilidades, asociadas o no asociadas a SIDA, determinaron ingresos como por ejemplo en casos de peritonitis bacteriana espontánea o hemorragia en pacientes con cirrosis o un cuadro sincopal por enfermedad del nodo sinusal.

Las infecciones representaron un sustrato relevante en este trabajo por varias razones: estuvieron presentes como causa originaria del ingreso en más de $80 \%$ de los casos, un hallazgo que es concordante con la literatura médica $^{8,11}$; se asociaron en algún momento de la evolución al desarrollo de shock séptico en cerca de la mitad de los casos; se expresaron como una panoplia de infecciones oportunistas y no oportunistas; y, en algunos casos como co-infecciones. Este perfil es importante debido al elevado requerimiento de recursos diagnósticos y terapéuticos cuando ingresan estos pacientes y la necesidad de buscar simultáneamente varios agentes infecciosos. Los equipos de salud, especialmente en el subsistema público muchas veces no cuentan con los recursos necesarios para lograr el diagnóstico definitivo lo que favorece el excesivo uso de terapias empíricas.

La mayor parte de las infecciones fueron pulmonares incluyendo casos por tuberculosis, $S$. pneumoniae, $P$. jiroveci (el agente individual más frecuente), CMV y cuadros sin microorganismos identificados. Este patrón es concordante con lo descrito previamente ${ }^{11}$. Aunque infrecuente, la neumonía por $\mathrm{CMV}$ ha sido reportada en pacientes con infección por VIH/SIDA y asociada como en este caso a un mal pronóstico ${ }^{15}$. Las infecciones mixtas, siempre con un compromiso pulmonar asociado y las neurológicas, ocuparon un papel importante dentro de las infecciones. Al respecto, no sólo los cuadros de toxoplasmosis estuvieron asociados a infecciones del SNC en la etapa SIDA, sino que también eventos de meningitis aguda asociada a primoinfección viral, tal como ha sido reportado previamente en nuestro medio ${ }^{16,17}$. La existencia de un caso de malaria grave revela la importancia de la exposición geográfica por turismo o razones laborales ${ }^{18}$. Aunque menos frecuente, las causas no infecciosas también estuvieron presentes, en parte explicadas por comorbilidades, postración o por linfoma asociado a SIDA. Un caso de síndrome de Guillaín Barré fue identificado en esta serie asociado a un bajo recuento de CD4 $\left(\sim 50 / \mathrm{mm}^{3}\right)$ que no tuvo respuesta a terapia con inmunoglobulina. Esta asociación ha sido descrita ${ }^{19}$.

Los ingresos a UCI estuvieron motivados principalmente por falla respiratoria, sepsis grave o shock séptico y compromiso neurológico, ya fuera en forma aislada o combinada. Estas causas son similares en proporción a lo registrado en otras latitudes en Latinoamérica ${ }^{11,20}$. En países desarrollados los ingresos por causa respiratoria han disminuido progresivamente y han aumentado las admisiones por co-morbilidades como co-infección por virus $\mathrm{C}$ de la hepatitis o complicaciones tardías de la infección por VIH en tratamiento ${ }^{21,22}$. La edad de los pacientes ha aumentado y la mortalidad se ha reducido progresivamente, en algunos centros a cifras bajo $10 \%{ }^{22}$.

Los hallazgos del examen físico fueron importantes para orientar la búsqueda del foco infeccioso y establecer la gravedad del cuadro séptico. Además, como se demuestra en este trabajo, permiten también predecir de algún modo la falla respiratoria y sospechar agentes involucrados.

En nuestro trabajo, la ausencia de TARGA, un recuento de linfocitos CD $4<250 / \mathrm{mm}^{3}$, la presencia de candidiasis oral o neumonía documentada por $P$. jiroveci se asociaron en el análisis univariado al desarrollo de falla respiratoria, rasgos compatibles con el riesgo de infecciones pulmonares potencialmente letales por $P$. jiroveci o CMV.

La mortalidad hospitalaria estuvo ligada al uso prolongado de fármacos vasoactivos. Este factor ha sido identificado con mayor mortalidad en otros trabajos ${ }^{11}$. 
Otras variables independientes asociadas a mortalidad hospitalaria descritos en la literatura incluyen sarcoma de Kaposi, recuento CD4 $\leq 50 / \mathrm{mm}^{3}$, pobre estado funcional, sepsis grave o shock séptico, puntaje APACHE II elevado, ventilación mecánica, falla cardíaca, tratamiento para tuberculosis o un bajo recuento de linfocitos CD4 en pacientes colonizados o infectados por agentes nosocomiales ${ }^{1,3,11,20,23,24}$.

Un hallazgo importante en esta investigación, es la baja sobrevida a mediano y largo plazo que tienen los pacientes que ingresa con un puntaje APACHE II elevado y que está en línea con otros predictores descritos como sepsis grave o shock séptico ${ }^{11,20}$. La sobrevida se estabiliza después de 18 meses, cifra cercana a los 12 meses reportada en otros trabajos $^{25}$. Desde la vereda opuesta, una mortalidad hospitalaria de $25 \%$ con una sobrevida que se estabiliza a los 18 meses indica que los esfuerzos terapéuticos con estos pacientes se justifican no sólo en el cuadro agudo sino que para los años venideros. Por otra parte, la declinación progresiva de la mortalidad en UCI observada en países desarrollados ${ }^{22}$, el impacto beneficioso aparente de una TARGA precoz ${ }^{26}$ y la estabilización del riesgo de muerte luego del egreso como se observó en este trabajo, son argumentos que justifican el ingreso de estos pacientes a una UCI a pesar del rechazo de algunos equipos médicos para adoptar tal medida ${ }^{27}$.

Un paciente falleció pocas horas después de recibir su primera dosis de TARGA con abacavir-lamivudinaefavirenz, en un cuadro compatible con una reacción de hipersensibilidad a abacavir o lamivudina y que cursó con hipotensión arterial y exantema. La probabilidad de desarrollar estas reacciones ante abacabir en pacientes que no portan el alelo HLA B*5701 es muy baja y por precaución se recomienda efectuar el análisis genómico antes de iniciar su uso ${ }^{28}$. No obstante, se han descrito algunos casos de reacciones graves por hipersensibilidad en pacientes HLA B*5701 negativos, incluso en las primeras horas ${ }^{29}$. Existe también la posibilidad de que esta anafilaxia pueda estar asociada a lamivudina ${ }^{30}$.

No existen consensos ni estudios de distribución aleatoria, prospectivos, que hayan definido el uso del TARGA en pacientes críticos. La prescripción precoz podría disminuir la mortalidad y morbilidad por la recuperación inmune pero también podría aumentar la morbilidad o mortalidad por efectos adversos, interacciones farmacológicas o el síndrome de reconstitución inmune ${ }^{31}$. Un estudio retrospectivo indicó que la mortalidad en pacientes con neumonía por $P$. jiroveci se redujo significativamente en aquellos pacientes que mantienen o inician su terapia en $\mathrm{UCI}^{32}$. Un estudio en Brasil detectó que el inicio o continuación de TARGA se asoció junto a otras variables independientes, a un menor riesgo de morir a los 6 meses de ingresar a una UCI (hazard ratio 0,5 ) y además observó que este efecto protector está ligado al inicio de
TARGA en los primeros cuatro días de ingreso a $\mathrm{UCI}^{26}$. En la misma línea, en Francia el inicio o continuación de TARGA fue un factor ligado a una menor mortalidad en UCI (odds ratio 0,28) y el inicio del TARGA a una menor mortalidad a los seis meses de seguimiento (OR 0,17) ${ }^{33}$. Otros estudios no han detectado variaciones significativas en la mortalidad entre grupos con y sin tratamiento ${ }^{31}$. Así, la evidencia retrospectiva sugiere una posible ventaja a corto o mediano plazo para el inicio precoz del TARGA en pacientes que ingresan a UCI. Para pacientes que ya están en terapia, algunos expertos recomiendan continuar ésta si hay éxito virológico y atender las posibles interacciones farmacológicas y ajustes por disfunción de órganos ${ }^{21}$. La alta letalidad de los pacientes que iniciaron TARGA en nuestros casos (5 de 7) y su inmediatez respecto al inicio de ella sugieren que su comienzo fue tardío o que estuvo más allá de las posibilidades médicas. Las reacciones adversas graves inesperadas forman parte de esta decisión.

Este trabajo tiene limitaciones que deben ser consideradas, incluyendo su reducido número de pacientes, lo que impidió encontrar otras asociaciones de importancia o efectuar análisis multivariado en algunos casos. Además, no todos los agentes infecciosos pudieron ser sistemáticamente estudiados lo que generó una proporción de infecciones sin etiología identificada. Por otra parte, los estándares de manejo pudieron haber variado en los nueve años incluidos en esta serie generando variaciones en la intensidad de estudio o de terapia. Finalmente, representan un solo centro y sus conclusiones no son necesariamente extrapolables a otros establecimientos.

En conclusión, los ingresos de pacientes con infección por VIH/SIDA a UPC siguen ocurriendo en nuestro país a pesar del acceso a terapia, incluyendo pacientes con diagnóstico conocido e indicación de recibir esta terapia, fragmento que explica cerca de $25 \%$ de esta serie subrayando a su vez un gran desafío de salud pública. El perfil de ingresos, mayoritariamente por causa infecciosa, es propio de países en desarrollo, contribuyendo a ello, la ausencia de quimioprofilaxis en una parte de ellos y a hospitalizaciones evitables. Se pudo también corroborar, la estrecha asociación entre ingreso por causa respiratoria y ausencia de TARGA, bajo recuento de linfocitos CD4 o candidiasis oral. Asimismo, al igual que en otras series, la mortalidad hospitalaria aparece ligada al uso de fármacos vasoactivos pero la mortalidad se mantiene en los meses siguientes, no estabilizándose hasta varios meses postegreso, siendo un score APACHE II elevado el factor que mejor indica un riesgo de morir durante la hospitalización o después del alta.

\section{Resumen}

Antecedentes y Objetivos: A pesar de la disponibilidad de la terapia viral de gran actividad (TARGA) en Chile 
el ingreso de pacientes con infección por VIH/SIDA a la Unidad de Pacientes Críticos (UPC) siguen ocurriendo. Se necesita mayor información en Chile sobre el perfil de estos pacientes, su mortalidad y el porcentaje de ingresos evitables. Método: Estudio observacional retrospectivo de pacientes adultos que ingresaron a la UPC en un hospital general durante 9 años. Resultados: En el período 2005-2013 se identificaron 32 pacientes que ingresaron a UPC. El 87,5\% estaba en etapa SIDA al ingreso, $53,1 \%$ sabía su diagnóstico, sólo 43,8\% recibía terapia TARGA y $16,6 \%$ quimioprofilaxis. Un $75,9 \%$ tenía un recuento CD4 $<200 / \mu \mathrm{L}$. La mayoría de los ingresos fue por una causa infecciosa $(84,4 \%)$ y $48,1 \%$ presentaron shock séptico. Por sistemas, los ingresos a UPC fueron liderados por falla respiratoria, compromiso neurológico, sepsis o una mezcla de ellos $(87,5 \%)$. Por análisis univariado, el ingreso por falla respiratoria se asoció a ausencia de HAART, candidiasis oral o un recuento $\mathrm{CD} 4<250 / \mu \mathrm{L}(\mathrm{p}<0,01)$. Ocho pacientes fallecieron en la primera hospitalización (25\%) y otros cinco en los meses siguientes al alta. El desenlace fatal en el hospital estuvo significativamente asociado al uso de fármacos vasoactivos por $\geq 7$ días (OR 16,5; IC 95 2,1-128 $\mathrm{p}<0,01)$. En el análisis multivariado, un score APACHE $\geq 18$ se asoció en forma independiente a fallecimiento en el hospital o post alta (OR 3,3 IC 95 1,1-10; $\mathrm{p}<0,05)$. Cuatro pacientes $(12,5 \%)$, tuvieron hospitalizaciones potencialmente evitables. Conclusiones: Las hospitalizaciones de pacientes con infección VIH a UPC siguen ocurriendo a pesar de la disponibilidad de TARGA en Chile, afectando a pacientes que desconocen su condición, no están en tratamiento o con profilaxis. Estos ingresos generan muertes prematuras, las que ocurren incluso después del alta en los pacientes más graves.

\section{Referencias bibliográficas}

1.- Vincent B, Timsit J F, Auburtin M, Schortgen F, Bouadma L, Wolff M, et al. Characteristics and outcomes of HIV-infected patients in the ICU: impact of the highly active antiretroviral treatment era. Intensive Care Med 2004; 30: 859-66

2.- Wolff M, Bustamante C, Bidart T, Dabanch J, Diomedi A, Northland R. Impacto de la terapia antirretroviral en la mortalidad de pacientes VIH+ chilenos: estudio caso-control MORTAR. Rev Med Chile 2000; 128: 839-45.

3.- Casalino E, Mendoza-Sassi G, Wolff M, Bedós J P, Gaudebout C, Regnier B, et al. Predictor of short-and long-term survival in HIV-infected patients admitted to the ICU. Chest 1998; 113: 421-9.

4.- Perelló R, Calvo M, Miró O, Castañeda M, Saubí N, Camón S, et al. Clinical presentation of acute coronary syndrome in HIV infected adults: a retrospective analysis of a prospectively collected cohort. Eur J Intern Med 2011; 22: 485-8.

5.- Sagredo S, Mancilla C, Estuardo N, Poniachik J. Falla hepática fulminante por virus hepatitis B en paciente coinfectado por VIH: Un desafío terapéutico. Caso clínico. Rev Med Chile 2011; 139: 1136-9.

6.- Pérez C, Muller C, Castillo Y, Mella S, Acuña G, Labarca J, et al. Neumonía por Pneumocystis carinii: factores predisponentes, manifestaciones clínicas y respuesta al tratamiento. Rev Chilena Infectol 1999; 16: 299-304.

7.- Andresen M, Boghero Y, Molgó M, Dougnac A, Díaz O. Necrolisis epidérmica tóxica. Terapia en UCI con inmunoglobulinas intravenosas en un caso. Rev Med Chile 2000; 128: 1343-8.

8.- Vera A, Pinochet R, Villamizar G, Cancino C,
Henríquez L, Cabib C. Ventilación mecánica en pacientes VIH+ en falla respiratoria aguda. Experiencia de una Unidad de Cuidados Intensivos de reciente creación. Rev Chil Med Intensiva 2006; 21: 67-72.

9.- Angus D, van der Poll T. Severe sepsis and septic shock. N Engl J Med 2013; 369: 840-51.

10.- Wolff M, Beltrán C. Programa de acceso ampliado a terapia antirretroviral en Chile, Plan AUGE y Cohorte chilena de SIDA: tres iniciativas complementarias. Rev Chilena Infectol 2004; 21: 281-4.

11.- Amâncio F F, Lambertucci J R, Cota G F, Antunes C M. Predictors of the short- and longterm survival of HIV-infected patients admitted to a Brazilian intensive care unit. Int J STD AIDS 2012; 23: 692-7.

12.- Gari S, Doig-Acuña C, Smail T, Malungo J R S, Martin-Hilber A, Merten S. Access to HIV/ AIDS care: a systematic review of socio-cultural determinants in low and high income countries. BMC Health Serv Res 2013; 13: 198.

13.- Ministerio de Salud de Chile. Informe Final. Estudio de carga de enfermedad y carga atribuible, Chile 2007. Disponible en: http:// epi.minsal.cl/epi/html/invest/cargaenf2008/ Informe\%20final\%20carga_Enf_2007.pdf

14.- Labarca E, Torres J, Cofré P. Sobrevida y calidad de vida dos años después de la Unidad de Cuidados Intensivos. Rev Chil Med Intensiv 2001; 16: 234-9.

15.- Erice A, Tierney C, Hirsch M, Caliendo A M, Weinberg A, Kendall M A, et al. Cytomegalovirus (CMV) and human immunodeficiency virus (HIV) burden, CMV end-organ disease, and survival in subjects with advanced HIV infection (AIDS Clinical Trials Group Protocol 360). Clin Infect Dis 2003; 37 : 567-78.

16.- Silva-Rosas C, Afani A, Fruns M,
Guzmán M A, Sepúlveda C. Meningitis en sujetos con infección por el virus de inmunodeficiencia humana. Neurología 1999; 14: 62-6.

17.- Sepúlveda C, Thompson L, Castrillón M A, Hurtado C. Infección primaria por VIH. Características clínicas y serológicas. Rev Med Chile 1995; 123: 74-80.

18.- Marks M E, Armstrong M, Suvari M M, Batson S, Whitty C J, Chiodini P L, et al. Severe imported falciparum malaria among adults requiring intensive care: a retrospective study at the hospital for tropical diseases, London. BMC Infect Dis 2013; 13: 118.

19.- Dalakas M C, Cupler E J. Neuropathies in HIV infection. Baillieres Clin Neurol 1996; 5: 199-218.

20.- Japiassú A M, Amâncio R T, Mesquita E C, Medeiros D M, Bernal H B, Nunes E P, et al. Sepsis is a major determinant of outcome in critically ill HIV/AIDS patients. Crit Care 2010; 14: R152.

21.- Huang L, Quartin A, Jones D, Havlir D V. Intensive care of patients with HIV infection. N Engl J Med 2006; 355: 173-81.

22.- Coquet I, Pavie J, Palmer P, Barbier F, Legriel S, Mayaux J, et al. Survival trends in critically ill HIV-infected patients in the highly active antiretroviral therapy era. Crit Care 2010; 14: R107.

23.- Greenberg J A, Lennox J L, Martin G S. Outcomes for critically ill patients with HIV and severe sepsis in the era of highly active antiretroviral therapy. J Crit Care 2012; 27 : 51-7.

24.- Ntusi N B, Badri M, Khalfey H, Whitelaw A, Oliver S, Piercy J, et al. ICU-associated Acinetobacter baumannii colonisation/infection in a high HIV-prevalence resource-poor setting. PLoS One 2012; 7 : e52452. 
25.- Turtle L, Vyakernam R, Menon-Johansson A, Nelson M R, Soni N. Intensive care usage by HIV-positive patients in the HAART era. Interdiscip Perspect Infect Dis 2011; 2011: 847835.

26.- Croda J, Croda M G, Neves A, De Sousa dos Santos S. Benefit of antiretroviral therapy on survival of human immunodeficiency virusinfected patients admitted to an intensive care unit. Crit Care Med 2009; 37: 1605-11.

27.- Corona A, Raimondi F. Critical care of HIV-infected patients: still a dilemma for Italian intensivists-results of a multicentre survey. Eur J Anaesthesiol 2010; 27 (4): 377-82.
28.- Pavlos R, Mallal S, Phillips E. HLA and pharmacogenetics of drug hypersensitivity Pharmacogenomics 2012; 13: 1285-306.

29.- Calza L, Rosseti N, Biagetti C, Pocaterra D, Colangeli V, Manfredi R. Abacavir-induced reaction with fever and severe skin rash in a patient tested human leukocyte antigenB*5701negative. Int J STD AIDS 2009; 20: 276-7.

30.- Kainer M A, Mijch A. Anaphylactoid reaction, angioedema, and urticaria associated with lamivudine. Lancet 1996; 348: 1519.

31.- Meybeck A, Lecomte L, Valette M, Van Grunderbeeck N, Boussekey N, Chiche A, et al. Should highly active antiretroviral therapy be prescribed in critically ill HIV-infected patients during the ICUstay? A retrospective cohort study. AIDS Res Ther 2012; 9: 27.

32.- Morris A, Wachter R M, Luce J, Turner J, Huang L. Improved survival with highly active antiretroviral therapy in HIV-infected patients with severe Pneumocystis carinii pneumonia. AIDS 2003; 17: 73-80

33.- Morquin D, Le Moing V, Mura T, Makinson A, Klouche K, Jonquet O, et al. Short- and long-term outcomes of HIV-infected patients admitted to the intensive care unit: impact of antiretroviral therapy and immunovirological status. Ann Intensive Care 2012; $2: 25$. 